\title{
Ganze Teile Zum kunsthistorischen Gattungsbegriff
}

\author{
Von Wolfgang KemP (Hamburg)
}

\section{ABSTRACT}

Die Frage nach den Bildgattungen wird heute von der Kunstwissenschaft mit einem Hinweis auf die historischen Aussagen über dieselben beantwortet. Mein Diskussionsbeitrag plädiert für eine Gattungstheorie, die sich stärker auf den Ursprung und die geschichtliche Eigenbewegung der Gattungen besinnt. Voraussetzung dafür ist die Anerkennung der Tatsachen, daß erstens am Anfang des Gattungsgeschehens die Aufteilung der Welt in bildförmige Themen steht und zweitens die so konstituierten Bildgattungen ihre Partialität zugleich anerkennen und überwinden. Sie erkennen das Ausschnitthafte ihrer Sicht an, indem sie eine Klasse von Gegenständen zu einem Seinsbereich umformulieren. Sie überwinden ihre Begrenzungen, indem sie Strategien der inneren Komplettierung entwickeln. So verstanden, als potenzierte Einheit aus Eigenem und Anderem, lassen sie sich auf die größte Herausforderung der Neuzeit ein, die Yirmiyahu Yovel das „Abenteuer der Immanenz“ genannt hat.

When it comes to questions of genre, arthistorians refer to the "sources", i.e. treatises from the $15^{\text {th }}$ century onwards. My contribution asks for a theory which focuses more closely on the origins and the internal historical development of genres. Two factors have to be reckoned with: firstly the original division of the world into painterly themes (like portrait, landscape, still-life etc.), and secondly the complex mechanism through which the various genres acknowledge their partial nature and try to surpass it at the same time. They realize their partiality by transforming a class of objects into a realm of being. They overcome their limits by bringing back the lost unity of the world in small, symbolic forms of compensation. Seen from this perspective, as complex unities of the particular and the other, genres face the greatest challenge of modern times, the "adventure of immanence", to use Yirmiyahu Yovel's words.

Im Grunde kann die kunsthistorische Gattungstheorie schon mit Aristoteles nicht mithalten. Dieser hatte die Dichtungsformen nach drei Kriterien unterschieden: nach den Kriterien der Darstellungsmittel (Vers, Prosa), der Gegenstände der Dichtung (hohe, mittlere und niedere Themen bzw. Personen) und der Modi der Darstellung (Rede der Personen, des Dichters etc.). Wenn wir den kunsthistorischen Gattungsbegriff auf die klassischen Gattungen der Malerei: Historie, Porträt, Landschaft, Stilleben etc. beschränken, dann haben wir nicht mehr anzubieten als das Gegenstandskriterium. Diese Tatsache hat die Kunstwissenschaft aus der komplexen und kontroversen Diskussion um die Begründung und Anwendbarkeit der Kategorie Gattung ausgeschlossen. Richtig interessant, denkt man, wird es erst, wenn modale und mediale Prinzipien ins Spiel kommen. Ist dies eine interessante Zeit der Gattungsdiskussion?

Die derzeitige Situation stellt sich in den Literaturwissenschaften so dar, daß die Zeiten des bemühten Anti-Substantialismus vorüber sind, der in Anlehnung 
an die Programmatik der Radikalmoderne die Relevanz von Gattungskonventionen verwarf - sei es im Namen der unverfügbaren Individualität des Kunstwerks (Croce), sei es als „Textualismus“ (Barthes) auf der Suche nach anderen Ebenen der Analyse und der Produktion, sei es in poststrukturalistischer Sorge um die Unabschließbarkeit aller Ordnungsversuche. Stattdessen ist eine Art Waffenruhe eingekehrt, die so verschiedene Geister wie E.D. Hirsch oder Frederic Jameson zusammengeführt hat, nicht unter dem Dach eines neuen Substantialismus, sondern jenes Konstruktivismus, der das Problem der Geltung von der eigenen Aussage auf die Aussagen der anderen oder auf die faktische Macht einmal etablierter Typen verschiebt. Gattungen, sagen sie, sagen viele Literaturwissenschaftler, funktionieren als soziale Normen, als literarische Institutionen, als sinngebender Rahmen. ${ }^{1,2}$

Im Fach Kunstgeschichte beobachten wir ebenfalls eine Ausweichbewegung vor dem, was in unschuldigeren Zeiten „das Wesen der Gattungen“ hieß. Die Mehrheit der auf diesem Gebiet tätigen Forscher bezieht geradezu reflexhaft alle Vorgänge der Gattungsgeschichte auf die Kunsttheorie und speziell auf die antike Poetik oder auf ekphrastische Texte des Altertums. ${ }^{2}$ Die nachantike, neuzeitliche Kunstgeschichte liest sich in ihrer Darstellung als ein gigantischer Nachholakt der verlorengegangenen Malerei des Altertums. Und jenseits der antiken Texte figuriert die Kunstliteratur seit Alberti als der Stichwortgeber und die Berufungsinstanz der Praxis. Was heißt, daß literarische Zeugnisse nicht etwa nur zum Verständnishorizont einer Zeit befragt, sondern als Verursacher von Gattungsgeschichte verantwortlich gemacht werden. Gombrich hat vermutlich mit diesem Reigen den Anfang gemacht, als er die Entstehung der Landschaftsmalerei im 16. Jahrhundert durch die Lektüre antiker Texte angeregt sah. ${ }^{3}$

Die Vorstellungen der Zeitgenossen können - um mit Reinhart Koselleck zu sprechen - ein Vetorecht ausüben, und sie sind selbstverständlich auch als Gegenstand eigenen Rechts zu studieren. Aber darum geht es hier nicht. Es wird auch niemand bestreiten, daß zwischen Theorie und Praxis Wechselwirkungen stattgefunden haben - die Frage ist, ob wir unsere Diskussionen über die Gat-

${ }_{1}$ Das neuere Schrifttum zum Thema literarische Gattungen kann hier nicht aufgelistet werden. Als nützlicher Überblick seien empfohlen: Karl Canvat, „Essai d'histoire de la notion de genre littéraire“, in: Les Lettres Roman LI, 1997, 187ff. (mit umfangreicher Bibliographie vor allem romanistischer Ausrichtung) und die Artikel „Gattung “, „Gattungsgeschichte“, in: Reallexikon der deutschen Literaturwissenschaft, Berlin $1997 \mathrm{ff}$.

2 Aus deutschsprachiger Sicht zu dieser Position Wilhelm Voßkamp, „Gattungen als literarisch-soziale Institutionen “, in: Walter Hinck (Hrsg.), Textsortenlehre-Gattungsgeschichte, Heidelberg 1977, $27 \mathrm{ff}$.

${ }^{3}$ Es ist in diesem Sinne konsequent, daß das anspruchsvollste Unternehmen zur Gattungsgeschichte fünf Quellenbände hervorgebracht hat - die Rede ist von der Reihe Geschichte der klassischen Bildgattungen in Quellentexten und Kommentaren, Berlin $1996 \mathrm{ff}$. 
tungen auf der Basis historischer oder gegenwärtiger Theorien weiterführen wollen. Gegen die erstgenannte Möglichkeit bzw. ihre Verabsolutierung zwei Argumente: ein historisches und ein systematisches.

1. In historischer Hinsicht sollte man von den Regularien der Kunstliteratur nicht zuviel erwarten, auch in Zeiten der normativen Ästhetik nicht. Nehmen wir eine der stärksten Vorgaben der akademischen Theorie, die bekannte Rangskala der Gattungen, die spätestens seit dem 17. Jahrhundert „in Kraft ist“. Es dürfte kaum einen europäischen Künstler gegeben haben, der nicht wußte, welche Bildgattungen zu suchen und welche eher zu meiden waren, welche man mit höheren Ansprüchen und dementsprechend besseren Chancen zu sozialer Anerkennung verband und welche nicht. Hat man sich daran gehalten? Zwei Antworten dürften schwer von der Hand zu weisen sein: Es sind nicht alle Künstler Historienmaler geworden. Und die sogenannten niedrigen Gattungen wie Stilleben und Porträt sind nicht in den Händen von Stümpern und Malknechten verkommen. Vielmehr hat in ihnen ein- und dieselbe künstlerische Intelligenz gewirkt und den Gattungsprozeß vorangebracht. „Tiefgreifende Änderungen in einer Gattung bleiben nicht ohne Einfluß auf die anderen, betreffen schließlich den gesamten Gattungszusammenhang. " ${ }^{4}$ Das ist so, weil es Gattung in der Einzahl nicht geben kann.

2. Das systematische Argument gegen eine Präjudizierung der Malerei durch die Theorie der Malerei ist ein sehr einfaches: Alle Anregungen „von außen“ müssen "nach innen“ vermittelt werden. Sie setzen nicht nur ein entsprechendes Interesse an ihnen voraus, sondern auch eine „Weise und Richtung der Objektivierung “, wie Cassirer gesagt hätte. Ohne, daß es ein Gattungsschema gäbe, könnten sie nicht wirksam werden. Diesem aber muß unsere Aufmerksamkeit gehören.

Die russischen Theoretiker, die nach dem Formalismus kamen, hatten sich mit einem ähnlichen Problem auseinanderzusetzen, mit der Erwartung nämlich, daß Gattungen vorgefertigte philosophische Konzepte oder zumindest geistesgeschichtliche Hauptanliegen realisieren. Sie verwahrten sich gegen diese Erwartung, indem sie aus der Not der Gattung, die Partialität heißt, die Tugend der Eigenart machten. Gattungen, so geht das Argument, finden auf ihre je eigene Weise zu ihren Aussagen, und diese sind nicht Verdoppelungen bereits existierender Konzepte im Reich der Ideen. „Jede Gattung besitzt bestimmte Prinzipien der Auswahl ... und ein bestimmtes Ziel und eine bestimmte Tiefe der

${ }^{4}$ Gottfried Boehm, Bildnis und Individuum. Über den Ursprung der Porträtmalerei in der italienischen Renaissance, München 1985, 251. Boehms Kapitel: „Gattung und Gattungen im historischen Prozeß “ ist m.E. der wichtigste Beitrag der Kunstwissenschaft zur Gattungsdiskussion. 
Durchdringung.“5 „Um erfolgreich schaffen zu können“, erklärte Pavel Medvedev, „muß der Künstler lernen, die Realität mit den Augen der Gattung zu sehen. Der Künstler muß jene Aspekte der Wirklichkeit erkennen, die dem gegebenen Genre entsprechen, er muß sie nach der Art dieses Genre ausdrücken, und er muß das Potential dieser Sichtweise ausbeuten, um etwas genuin Neues und Wertvolles auszudrücken. “6 Das „Auge der Gattung " aber sieht, bevor es lesen kann. Was nicht heißt, daß es nicht theoretisch ist.

Diese Diskussion abschließend ließe sich sagen: Die Kunstgeschichte ist nicht nur als Quellenkunde gefragt. Wer später kommt und sich mit wissenschaftlichem Interesse den historischen Gegenständen nähert, braucht eigene Begriffe und Instrumentarien der Analyse. Wenn das nicht so undialektisch wäre, müßte man wie seinerzeit Wölfflin die Stärkung der „begrifflichen Forschung “ gegenüber der historischen einfordern.

Nach dem Motto: „Was man nicht verbergen kann, muß man betonen“ sollte die Kunstwissenschaft das scheinbar triviale Gegenstandskriterium selbstbewußt annehmen und zunächst einmal sagen, daß durch die Gattungsmalerei eine vollständige Aufteilung der Welt in die den Menschen angehenden Seinsbereiche stattfindet. Die Gattungen behandeln das menschliche Verhältnis zur Geschichte (Historienbild), zum praktischen, alltäglichen Leben (Genre), zur Natur (Landschaft), zu sich als Individuum (Porträt), zur Objektwelt (Stilleben) etc. Diese Aufteilung der Welt bedeutet eine grundstürzende Umorientierung. Die Transzendenz, die als Thema und als organisierendes Prinzip die Kunstproduktion bis dahin bestimmt hatte, wird in die Gattungsmalerei mit einbezogen (als religiöse Historienmalerei, als religiöse Symbolik in Stilleben etc.), aber sie formiert nicht mehr die Einrichtung dieser Welt. Für die Malerei beginnt, was Yovel das „Abenteuer der Immanenz“ genannt hat.

Während die christliche Kunst der 1000 Jahre von 400 bis 1400 ihre Leitform in der Bildsumme hatte, die dem Teilbild nur eine untergeordnete Funktion bei der Explikation des Ganzen einräumte, ist das Einzelbild, das eine ähnlich führende Position seitdem innehat, der Träger der Gattungsmalerei. Beide, Tableau und Gattung, sind miteinander geworden und aneinander gewachsen. Medialität erzeugt Modalität und umgekehrt, d.h. wir dürfen doch noch einmal zu Aristoteles aufschließen und den Gehalt der historischen Voraussetzungen und Folgen, die zur scheinbar simplen Gegenstandskategorisierung gehören, wichtig machen. Gottfried Boehm hat den gemeinsamen Prozeß von Medium und Modus unter das Stichwort „Individualisierung “ gebracht: „Dem neu-

5 Pavel Medvedev, The Formal Method in Literary Scholarship: A Critical Introduction to Sociological Poetics, Cambridge (Mass.) 1985, 276 (zuerst 1928). Zur Autorschaft und allgemein zu Fragen der literarischen Gattungslehre bei Medvedev und vor allem bei Bakhtin siehe Gary Saul Morson, Caryl Emerson, Mikhail Bakhtin. Creation of a Prosaics, Stanford 1990, $272 \mathrm{ff}$.

${ }^{6}$ Medvedev (Anm.5). 
en Bedürfnis, einen Ausschnitt gesehener Natur zum Thema zu setzen, verbündet sich die Fähigkeit, darin einen Sinn darzulegen, den Teil als Einheit zu erfassen. Dieser Prozeß der Stilisierung leitet das Auge des Betrachters, ermöglicht ihm, die Beschränkung der jeweiligen Naturansicht doch als ein Bild zu lesen, in dem sich das Dargestellte und die Formstruktur wechselseitig individualisieren. Das Landschaftsbild zeigt künstlerisch individualisierte Natur. In ihm ist das Dilemma zwischen der Kontingenz einer begrenzten Sicht auf Natur und dem Ordnungscharakter von Natur geschlichtet “"?

Diese Überlegungen haben wichtige Konsequenzen für den analytischen Umgang mit Gattungsmalerei. Die Vermutung wird nicht zum ersten Mal ausgesprochen, daß der skizzierte Traditionsbruch, der mit der Neuzeit eintritt, allerlei kompensatorische Schritte ausgelöst hat. Das Teilen und das den Teil gelten lassen, beides will offensichtlich gelernt und ausgehalten sein. Worauf uns keine historische Kunsttheorie vorbereitet, ist z.B. die Tatsache, daß Gattungsmalerei oft „unrein“ ist. In Verlängerung des oben Gesagten könnte man so das Gattungsgeschehen als unabgeschlossene Auseinandersetzung mit dem Verlust der Totalität begreifen. Gattungsmischung wäre eine Antwort; eine andere könnte man als innere Komplettierung bezeichnen. In letzterem Sinne läßt sich das von Yovel in seinem Spinoza-Buch ausgegebene Stichwort „Abenteuer der Immanenz" ${ }^{8}$ zweifach verstehen: als Eroberung der endlichen Dinge in ihrer Vielfalt und Eigenart und weiterhin als Versuch, dem Problem der verlorenen Totalität durch Weisen interner Komplettierung zu begegnen. Klassische Figuren solcher "Wiederholung" des Abgetrennten sind die Öffnung auf ein anderes Weltsegment (Fenster im Hintergrund mit Blick auf Landschaft), die Einlage (Manets Stilleben im Dejeuner sur l'herbe), die Einbettung im Bild, die Substitution (bei van Gogh kann ein Interieur ein Selbstporträt sein) etc.

Das Gesetz einer künstlerischen Gattung heißt danach, eine Welt eingrenzen und dann das Ausgegrenzte in gattungsförmig anverwandelter Form wieder zuzulassen. Die Gattung ist eine komplementäre Ganzheit oder Gestalt, eine potenzierte Einheit aus dem Eigenen, dem Nächsten und dem anderen. Von besonderer Wichtigkeit ist, daß wir die Formulierung ,in gattungsförmig anverwandelter Form “ immer mitdenken. Zu lernen, etwas unter Bedingungen, „mit den Augen “ einer Gattung zu zeigen und wahrzunehmen, ist neben der Teil-alsGanzes-Wahrnehmung der zweite entscheidende Schritt, den neuzeitliche Malerei über die mittelalterliche hinaus macht. Die Integration des Ausgegrenzten bedeutet, daß das gattungsmäßig Begrenzte sich einen Verweisungskontext zulegt, sich in einen Horizont stellt.

Damit sind wir, von außen kommend, schließlich da angelangt, wo wir fragen müssen, wie die einzelne Gattung mit ihrem allereigensten Gegenstand um-

7 Boehm (Anm. 4), 253.

8 Yirmiyahu Yovel, Spinoza. Das Abenteuer der Immanenz, Göttingen 1996. 
geht. In Abwandlung des zuletzt Gesagten darf gelten, daß die Gattung eine Klasse von Gegenständen zu einem Seinsbereich, zu einer Weise des In-derWelt-Seins umformt. Ein Interieur ist erst dann ein Interieur, wenn alle Gegenstände, auch diejenigen, die in den Zuständigkeitsbereich anderer Gattungen fallen, unter den Gesetzen des Innenräumlichen erscheinen, das ist der Unterschied zwischen einer „Frau am Spinett“ und einem „Interieur mit Frau am Spinett". Seinsbereiche aber etablieren sich im Spannungsfeld von Gegensätzen, deren je neue Belegung den Prozeß der Gattungsgeschichte am Leben hält. Produktive Gegensätze können sein die Hier-Dort-Opposition in der Landschaftsmalerei, das Verhältnis von Innen und Außen im Interieur, von Privat und Öffentlichkeit beim Genre, von Ich und Du und von Individuum und gesellschaftlichem Wirkfeld beim Porträt. Doch können dies nur vorläufige Angaben zu einer systematischen Bestimmung kunsthistorischer Gattungen sein. 